This is a provisional PDF only. Copyedited and fully formatted version will be made available soon.
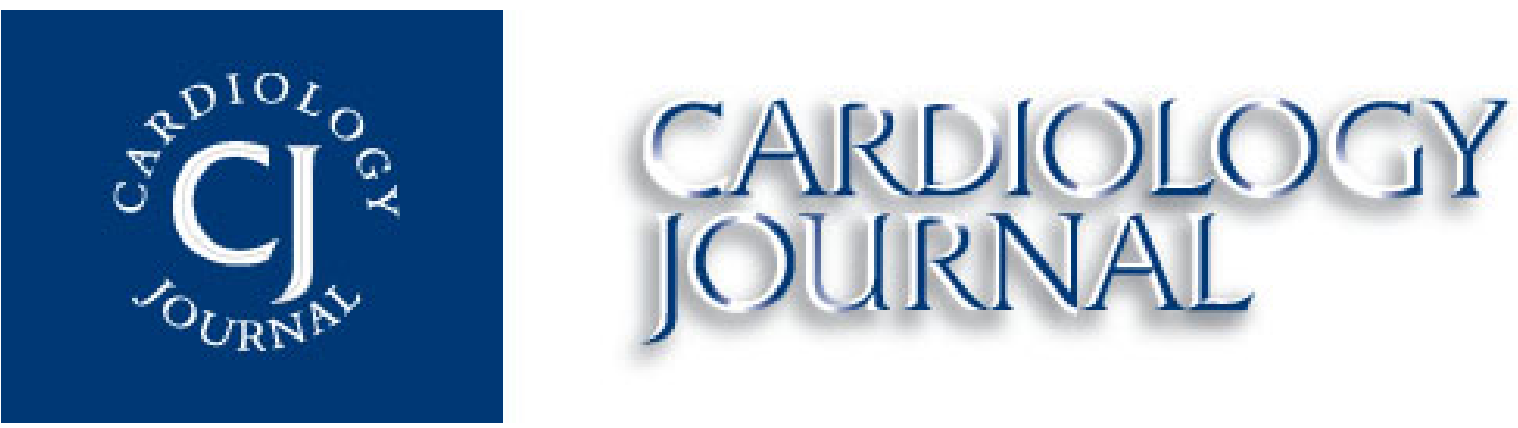

ISSN: 1897-5593

e-ISSN: 1898-018X

\title{
Comparison of the protein acetylome of endothelial cells upon shear flow and resveratrol treatment
}

Authors: Wun-Rong Lin, Chung-Jung Liu, Yaw-Syan Fu, Fu-An Li, Bin Huang

DOI: $10.5603 /$ J.a2020.0029

Article type: Original articles

Submitted: $2019-11-15$

Accepted: $2020-02-20$

Published online: 2020-02-25

This article has been peer reviewed and published immediately upon acceptance.

It is an open access article, which means that it can be downloaded, printed, and distributed freely, provided the work is properly cited.

Articles in "Cardiology Journal" are listed in PubMed. 


\section{Comparison of the protein acetylome of endothelial cells upon shear flow and resveratrol treatment}

Running title: Acetylome regulated by shear flow and resveratrol

Wun-Rong Lin ${ }^{1,2,3}$, Chung-Jung Liu ${ }^{4,5}$, Yaw-Syan $\mathrm{Fu}^{6,7}, \mathrm{Fu}-\mathrm{An} \mathrm{Li}{ }^{8}$, Bin Huang ${ }^{5,6,9,} 10$

${ }^{1}$ Department of Urology, MacKay Memorial Hospital, Taipei, Taiwan.

${ }^{2}$ Mackay Junior College of Medicine, Nursing, and Management, Taipei, Taiwan

${ }^{3}$ Department of Medicine, Mackay Medical College, New Taipei City, Taiwan

${ }^{4}$ Division of Gastroenterology, Department of Internal Medicine, Kaohsiung Medical University Hospital, Kaohsiung, Taiwan

${ }^{5}$ Regenerative Medicine and Cell Therapy Research Center, Kaohsiung Medical University, Kaohsiung, Taiwan

${ }^{6}$ Department of Biomedical Science and Environmental Biology, College of Life Science, Kaohsiung Medical University, Kaohsiung, Taiwan

${ }^{7}$ Department of Basic Medical Science, Xiamen Medical College, Xiamen, China

${ }^{8}$ Institute of Biomedical Sciences, Academia Sinica, Taipei, Taiwan

${ }^{9}$ Department of Medical Research, Kaohsiung Medical University Hospital, Kaohsiung, Taiwan

${ }^{10}$ Department of Biological Sciences, National Sun Yat-sen University, Kaohsiung, Taiwan

Address for correspondence: Bin Huang, PhD, Department of Biomedical Science and Environmental Biology, College of Life Science, Kaohsiung Medical University, No. 100, Shihchuan 1st Rd., San Ming District, Kaohsiung 80708, Taiwan, tel: 886-7-3121101 ext. 2704, fax: 886-7-3227508, e-mail: huangpin2@yahoo.com.tw 


\begin{abstract}
Background: Posttranslational acetylation/deacetylation known as the acetylome is important in regulating protein activity. Shear flow (SF) and resveratrol (RSV) are two stimuli that represent physical and chemical signals separately. The acetylome co-regulated by these two stimuli remain unclear.
\end{abstract}

Methods: Human umbilical cord vein endothelial cells (HUVECs) were subjected to either SF of 12 dynes $/ \mathrm{cm}^{2}$ or $10 \mu \mathrm{M}$ RSV. The purified acetylated peptides were labeled by isobaric tags for relative and absolute quantitation (iTRAQ) analysis. The signaling cascades of the identified acetylome were predicted by ingenuity pathway analysis (IPA). Coimmunoprecipitation was applied to confirm the acetylation status of the proteins.

Results: Five groups of proteins showed an increased acetylation upon SF and RSV treatment. After algorithm, 628 proteins with increased acetylation and 22 proteins with decreased acetylation were identified in the SF acetylome. For the acetylome regulated by RSV, 145 proteins with increased acetylation and 23 proteins with decreased acetylation were identified. These two acetylomes were compared, and 129 proteins with increased acetylation and 2 proteins with decreased acetylation were co-regulated by both SF and RSV treatments. IPA analysis showed that this co-regulated acetylome was involved in a heat shock response, and the signals of eNOS, STAT3, JAK/STAT and ERK/MAPK. Co-immunoprecipitation analysis further confirmed the acetylated status of mitochondrial HSP60 and mitochondrial citrate synthase.

Conclusions: This study indicated that a physical signal is more complicated than a chemical signal in the case of acetylome. The co-regulated proteins are worthy of further study in discussion of the synergetic effect between a physical and a chemical signal in cardioprotection.

Key words: acetylome, shear flow, resveratrol, iTRAQ, HSP60, ATP-citrate synthase 


\section{Introduction}

Protein acetylation is known as a posttranslational modification that can alter histoneDNA interactions and therefore regulates gene expression. A recent study show that nonhistone protein deacetylation has an important role in triggering physiological responses [1]. Acetylation at lysine (K) 221 and K310 promotes the activity of NF- $\kappa B$ and downstream

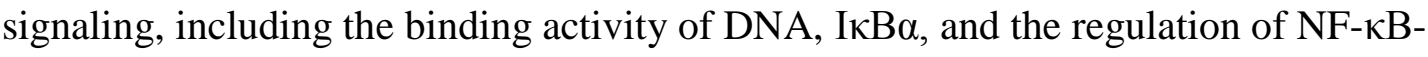
mediated inflammatory responses [2]. In endothelial cells (ECs), SIRT1 and SRT3 can modulate the function of endothelial nitric oxide synthase (eNOS), p53, angiotensin II (Ang II) type 1 receptor (AT1R), and forkhead box O (FOXO) which promotes cardiovascular homeostasis [3]. SIRTs proteins are known as deacetylases that remove the acetyl group from the lysine residue. In this way they regulate calorie restriction-induced anti-aging in cardiac myocytes [4]. Vascular endothelial growth factor receptor 2 (VEGFR2) is acetylated at four lysine residues forming a catalytic domain for tyrosine kinase, which indicates that posttranslational acetylation is a critical mechanism for affecting the VEGFR2 function [5]. Concurrent with these findings, the protein acetylome is of substantial importance in the adaptation of cells for different physiological functions.

Shear flow (SF) is a mechanical force that improves the function of ECs under diverse risk factors. ECs exposed to laminar SF prevent the progression of atherosclerosis [6]. Shear force can induce the production of hydrogen sulfide $\left(\mathrm{H}_{2} \mathrm{~S}\right)$ that can activate the nitric oxide (NO) signaling cascade in endothelial cells [7]. Laminar SF for at least 4 hours can activate SIRT1 and promotes autophagy by removing ROS, which is thought to be important in maintaining endothelial mitochondrial function $[8,9]$. In regions of disturbed laminar SF, irregular SF increases the risk of inflammation and oxidative injury in ECs [10]. Resveratrol (RSV) was the first natural polyphenolic compound discovered that regulates protein acetylation as well calorie restriction by stimulating SIRTs [11]. RSV can activate SIRT1 to 
regulate the acetylation of endothelial proteins that provide cardioprotection [12]. RSV can also activate SIRT3 and then improves cardiac function through the transformation of the growth factor- $\beta /$ Smad3 pathway [13]. Protein acetylomes involved in endothelial homeostasis that are co-regulated by physical or chemical signals are, therefore, providing insights in its mechanisms of action as well as possible cures for cardiovascular diseases.

For detecting protein acetylation, the western blot-based methodology was developed [14]. Isobaric tags for relative and absolute quantitation (iTRAQ) provide a labeling method used in shotgun quantitative proteomics coupled with tandem mass spectrometry [15]. It is known that the acetylome can either be regulated by SF or RSV $[12,16]$. To further elucidate the mechanisms of acetylome co-regulation, both physical stimulation from SF and chemical stimulus by RSV were studied. The tryptic peptides were labeled by iTRAQ and then subjected to LC-Q-Orbi MS/MS to calculate the expression levels of acetylated peptides. The changes of acetylated lysine residues can be determined simultaneously. Immunoprecipitation with anti-acetyl lysine was further applied to further identify the acetylome by tandem mass spectrometry. These acetylomes can provide valuable information about the protective mechanisms regulated by physical and chemical signals in endothelial cells.

\section{Methods}

HUVECs culture and treatment

Human umbilical vein endothelial cells (HUVECs) were purchased from Thermo Fisher Scientific Inc. (Thermo Fisher Scientific, Waltham, MA, USA). HUVECs were cultured in M199 medium (Thermo Fisher Scientific) supplemented with 20\% (v/v) fetal bovine serum (FBS), $10 \%$ (v/v) EBM-2 growth medium (Lonza, MD, USA), $100 \mathrm{U} / \mathrm{mL}$ penicillin/streptomycin, and then incubated in a growth chamber at $37^{\circ} \mathrm{C}$ with $\mathrm{CO}_{2}(5 \%$, v/v). Glass slides were coated with collagen prior to being seeded with HUVECs $\left(1 \times 10^{3}\right.$ cells $/ \mathrm{cm}^{2}$ ). After incubation in a growth chamber for 24 hours, the HUVECs were either 
harvested (static treatment) or exposed to SF with 12 dynes $/ \mathrm{cm}^{2}$ for 1 hour in an SF device. For RSV treatments, HUVECs were incubated with $10 \mu \mathrm{M}$ RSV (Tocris Bioscience, MN, USA) for 1 hour.

Cell lysis and protein extraction

The HUVECs were washed with $1 \times$ PBS buffer and then lysed with lysis buffer: HEPES (250 mM, pH 7.7), EDTA (1 mM), neocuproine (0.1 mM), and CHAPS (0.4\%, w/v). The protein concentrations were determined using the BCA assay reagent (Thermo Fisher Scientific).

In-solution trypsin digestion and iTRAQ labeling

The extracted proteins $(100 \mu \mathrm{g})$ were digested with trypsin (In-Gel Tryptic Digestion kit, Thermo Fisher Scientific) for 4 hours at $37^{\circ} \mathrm{C}$. The resulting tryptic peptides were labeled with the iTRAQ 4-plex reagent as follows: iTRAQ 114 for control (static treatment), iTRAQ 115 for SF treatment (12 dynes/cm ${ }^{2}, 1$ hour) and iTRAQ 116 for RSV treatment (10 $\mu \mathrm{M}, 1$ hour) according to manufacturer instructions (Applied Biosystems, Foster City, CA, USA). Labeling efficiency was evaluated with a Mascot search where respective isobaric modifications were set as variable modifications on the $\mathrm{N}$-terminus with lysine. The labeled peptides were then mixed and subsequently analyzed.

Liquid chromatography tandem-mass spectrometry (LC-MS/MS) analysis

The peptide sample mixed from treatments were preliminary fractionated by a cationexchange chromatography. Briefly, samples were reconstituted in $25 \%(\mathrm{v} / \mathrm{v})$ acetonitrile and 5 $\mathrm{mM} \mathrm{KH}_{2} \mathrm{PO}_{4}$ and loaded onto a PolySULFOETHYL A column (PolyLC, Columbia, MD, USA) in a high-performance liquid chromatography (HPLC) system (Agilent Technologies, Palo Alto, CA, USA). Separated peptides were eluted within a linear gradient of 0-350 mM 
$\mathrm{KCl}$ buffer: $5 \mathrm{mM} \mathrm{KH} \mathrm{KO}_{4}$ in $25 \%$ (v/v) acetonitrile, $\mathrm{pH}$ 2.7. A split-flow configuration of HPLC was used for online nanoLC separation and was coupled to an Orbitrap mass spectrometer (Thermo Fisher Scientific). The peptide samples were then injected into a homemade capillary trap column $(2 \mathrm{~cm} \times 100 \mu \mathrm{m}$ i.d., packed with Magic C18 AQ reversedphase material, $5 \mu \mathrm{m}, 200 \AA$, Michrom BioResources, Auburn, CA, USA). Then they were separated in a $13 \mathrm{~cm} \times 75 \mu \mathrm{m}$ i.d. capillary column $(10 \mu \mathrm{m}$ electrospray tip, packed with Magic C18AQ, $5 \mu \mathrm{m}, 100 \AA$ ). To identify iTRAQ-labeled peptides, mass spectra were acquired in the positive ion mode at a selected mass range of $350-1600 \mathrm{~m} / \mathrm{z}$. The mass spectrometric device was run in a top-ten configuration with one MS scan followed by 10 MS/MS scans. The acquisition of MS/MS spectra was operated in parallel mode, allowing accurate mass measurements of precursors in an Orbitrap. This was concurrent with the acquisition of a data-dependent collision-induced dissociation (CID) MS/MS of the five most intense precursor ions in the ion trap, followed by five higher-energy collisional dissociation (HCD) MS/MS scans on the same precursors. The precursor ions of peptides were selected with an isolation window of 2.0 Da, and a target value of $1 \times 10^{6}$. Dynamic exclusion was implemented with a repeat count of 1 and exclusion duration of $180 \mathrm{~s}$. The NCE was set to $35 \%$ for CID, and $60 \%$ for HCD to facilitate reporter ion generation.

Data analysis

Protein identification, and relative abundance quantification were performed using Proteome Discoverer v.1.3 (Thermo Fisher Scientific). The CID and HCD raw spectra were extracted and searched against the forward and decoy human Swiss-Prot database using the Proteome Discoverer with MASCOT search algorithm (Matrix Science, Boston, MA, USA). The search parameters used were as follows: peptide ion mass tolerance of 100 ppm; CID and HCD fragment ion mass tolerance of 0.8 Da and 0.1 Da, respectively; 2 missed trypsin cleavages; carboxamidomethylation of cysteine, variable oxidation of methionine, acetylation 
of lysine, deamidation of asparagine and glutamine; iTRAQ labeling of the N-termini of peptides and lysine side chain residues; and a false discovery rate $<1.0 \%$. In the CID-HCD dual scan configuration, peptide identification was obtained from the CID scan. Quantitation based on iTRAQ reporter ions was accomplished with a peptide mass tolerance of $20 \mathrm{ppm}$. The iTRAQ reporter ion intensities were extracted from the HCD scans and mapped to the same precursor ion identified from the corresponding CID scan. Thereafter, all peptide ratios were normalized against the median protein ratio.

Immunoprecipitation (IP)

Protein G beads (Millipore, Billerica, MA, USA) were incubated with anti-acetylation monoclonal antibody (BD Biosciences, San Jose, CA, USA) for 10 min at room temperature to generate a bead-antibody conjugate according to user guidelines. Four hundred micrograms of extracted proteins were incubated with antibody-linked beads at $4{ }^{\circ} \mathrm{C}$ overnight. The beads were finally resuspended in $40 \mu \mathrm{L}$ of sample buffer [Tris- $\mathrm{HCl}(62.5 \mathrm{mM}, \mathrm{pH} 6.8), \mathrm{SDS}$ (3\%, w/v), 2-mercaptoethanol (5\%,v/v), glycerol $(10 \%, v / v)]$ to elute the acetylated proteins.

Western blot

Forty micrograms of cell lysate or one microgram of acetylation-purified proteins (after IP) with various treatments were mixed with sample buffer [Tris- $\mathrm{HCl}(62.5 \mathrm{mM}, \mathrm{pH} 6.8)$, SDS (3\%, w/v), 2-mercaptoethanol (5\%, v/v), glycerol (10\%, v/v)], and then separated by SDS-PAGE. The gel was transferred to the PVDF membrane (Millipore) and immunoblotted with antibodies: HSP60 (1:1000, BD, Biosciences), HSP90 (1:1000, BD, Biosciences), antiacetyl lysine monoclonal antibody (1:1500; Cell Signaling Tech. Beverly, MA, USA), citrate synthase (GeneTex, Barcelona, Spain), and $\beta$-actin (1:3000, Millipore). The membranes were visualized with the SuperSignal West Femto Maximum Sensitivity Substrate (Thermo Fisher Scientific) and scanned using a digital scanner (Microtek International Inc., Hsinchu, 
Taiwan), then, the density was calculated by Progenesis Samespots v2.0 software (NonLinear Dynamics, Newcastle, UK).

\section{Results}

The HUVECs treated either with SF or RSV $(10 \mu \mathrm{M})$ were subjected to iTRAQ-labeling shotgun proteomics analysis as shown in Figure 1A. In western blotted analysis with antiacetyl lysine antibody, except for protein group f, the else proteins from group a to group e all showed a significant increase in acetyl modification in both SF and RSV treatments (Fig. 1B, C).

In iTRAQ-labeled shotgun proteomic analysis, tryptic peptides from the control (iTRAQ 114), SF (iTRAQ 115) and resveratrol (iTRAQ 116) treatments were subjected to LC-MS/MS analysis. Totally, 628 proteins showed an up-regulated acetylation and 22 proteins showed a down-regulated acetylation under the SF treatment. As for RSV treatment, 145 proteins showed an up-regulated acetylation and 23 proteins showed a down-regulated acetylation. There were 129 proteins with up-regulated acetylation and 2 proteins with down-regulated acetylation co-regulated either by SF or RSV treatments (Fig. 2A). Ingenuity pathway analysis (IPA) of the 129/2 acetylated/deacetylated proteins co-regulated by both treatments showed that these proteins are involved in signaling pathways, including heat a shock response, eNOS signaling, STAT3 pathway, JAK/STAT signaling and ERK/MAPK signaling cascades (Fig. 2B).

In addition to identifying the acetylated proteins, eight representative proteins with significant increases in acetylation level, and possible acetylated lysine residues between SF and RSV treatments were indicated (Fig. 3). To confirm the acetylation status of proteins identified by mass spectrometry, antobodies of heat shock protein 90 (HSP90), heat shock protein 60 (HSP60) and ATP-citrate synthase were applied in the IP assay. The western blots of whole cell lysate (before IP) and the eluents (after IP) were compared (Fig. 4A). The 
acetylation levels of HSP90 (Ac-HSP90) showed no statistical significance between treatments. As for HSP60, the acetylation levels were increased upon both SF and resveratrol treatments. Interestingly, the acetylation of ATP-citrate synthase (Ac-ATP-citrate synthase) can only be promoted by SF (Fig. 4B).

\section{Discussion}

A comparison of the protein acetylome regulated by physical (SF) and chemical (RSV) signals has rarely been investigated, according to available research. In this study, the SF signal induced 628/22 protein's acetylation/deacetylation, while the RSV signal induced $145 / 23$ proteins acetylation/deacetylation. This indicates that the physical signal can induce more signaling pathways in cellular responses when compared with the chemical signal. The IPA analysis showed that these co-regulated proteins can promote the production of nitric oxide, heat shock response, STAT3 and ERK signaling [7, 17]. Separately induced acetylation by SF and RSV were discussed in the literature $[16,17]$. According to a prediction of IPA analysis, the acetylation of mitochondria heat shock protein 60 (HSP60) can be co-upregulated by two treatments. HSP60 is a chaperone implicated in mitochondrial protein transportation and macromolecular assembly [18]. In endothelial cells that were stressed by oxidized LDL or SF, the expression of HSP60 on the cell surface is enhanced and triggers effects on auto-antigen and thus, acts as a proatherosclerotic molecule [19]. SF with 30 dynes $/ \mathrm{cm}^{2}$ for 12 hours induces the expression of HSP60 [20]. Acetylation can regulate the HSP60 complex assembly and cell autophagy after posttranslational modification [21]. In this study, SF and RSV treatments induced the acetylation of HSP60 at K156, K157, K352, K359 and K405 residues. The effects of acetylation of these lysine residues, particularly the structure of the catalytic site, and the enzymatic activity of HSP60 should be further studied by point mutation.

ATP-citrate synthase, also known as ATP-citrate lyase (ACLY), is a transferase 
involved in the TCA cycle for the production of ATP. For the reason that acetyl-CoA is a key index in evaluating the efficiency of cell metabolic regulation, the regulation of its enzymatic activity is interesting. ACLY can supply NADPH requirements for NO and ROS production during an inflammatory response [22]. As a result, ACLY could be a new biomarker for predicting the inflammation status. In addition to inhibited endothelial cell metabolism via KLF2-mediated repression of PFKFB3, SF is also known to regulate acetyl CoA [23]. In this study, the putative acetylation on residues of K58, K64, K148, K177, K259, K538, K554, K914, K1010 and K1018 identified by LC-MS/MS (Fig. 3) were only observed upon SF treatment but not in RSV treatment (Fig. 4B). This implied that ACLY is much more susceptible to physical signals.

HSP60 and ACLY are two proteins located in mitochondria which indicate that SF and RSV can substantially regulate mitochondrial function. The acetyl modification of mitochondrial proteins, particularly during deacetylation are well known to be important for mitochondrial biogenesis and activity [4]. However, it took a long time for SF (at least $4 \mathrm{~h}$ ) or RSV (at least $24 \mathrm{~h}$ ) to induce SIRTs-mediated protein deacetylation [9, 24]. Different to the protein deacetylation, a conflicting result with an enhanced protein acetylation was observed in the present short term (1 h) SF and RSV treatments. Such early responsive acetylomes may be responsible for the most fundamental mechanisms of physical and chemical signals in regulating SIRTs activity. More importantly, how these acetylated/deacetylated lysine residues can affect the protein activity of HSP60 and ACLY could be further investigated by the point mutation of lysine residue.

In the human body, blood shear force is variable from smaller than $1 \mathrm{dyne} / \mathrm{cm}^{2}$ to higher than 80 dynes/cm [2, 25]. Turbulent flow-induced irregular shear force always occurred in the regions of aortic arch, branches, and also the aortic valve. Therefore, the risks of plaque formation, stenosis and atherosclerosis are much higher. According to the present study, the correlations of these acetylomes, including the acetylations of HSP60 
and ACLY to cardiovascular disease can be investigated in ruptured thrombotic samples from a myocardial infarction patient. The possible medical solutions of patients with over curvature aorta or limbs with low blood shear flow might be proposed.

\section{Conclusions}

According to available research, acetylomes regulated by SF or RSV were first compared in this study. These acetylomes were involved in a heat shock response, eNOS signaling, STAT3 pathway, JAK/STAT pathway and ERK/MAPK pathway provide a new target for further understanding the correlation between acetylome and endothelium homeostasis.

\section{Acknowledgements}

This work was supported by the Kaohsiung Medical University "Aim for the Top Universities Grant” KMU-TP105G00, KMU-TC108A02. NSYSU-KMU JOINT RESEARCH PROJECT (\#NSYSUKMU 105-P032 and \#NSYSUKMU 106-P009). We are grateful to the core facility laboratories of the Institute of Biomedical Sciences, Academia Sinica and CRRD of Kaohsiung Medical University for their mass spectrometric analyses, and also the kind support from Mr. Wen-Kuo Hsieh and Dr. Wen-Chiuan Tsai from National Defense Medical Center. We acknowledge critical reading of the manuscript by Dr. Hans-Uwe Dahms.

Conflict of interest: None declared 


\section{References}

1. Narita T, Weinert BT, Choudhary C, et al. Functions and mechanisms of non-histone protein acetylation. Nat Rev Mol Cell Biol. 2019; 20(3): 156-174, doi: 10.1038/s41580-018-0081-3, indexed in Pubmed: 30467427.

2. Huang Bo, Yang XD, Lamb A, et al. Posttranslational modifications of NF-kappaB: another layer of regulation for NF-kappaB signaling pathway. Cell Signal. 2010; 22(9): 1282-1290, doi: 10.1016/j.cellsig.2010.03.017, indexed in Pubmed: 20363318.

3. D'Onofrio N, Servillo L, Balestrieri ML. SIRT1 and SIRT6 Signaling Pathways in Cardiovascular Disease Protection. Antioxid Redox Signal. 2018; 28(8): 711-732, doi: 10.1089/ars.2017.7178, indexed in Pubmed: 28661724.

4. Zullo A, Simone E, Grimaldi M, et al. Sirtuins as mediator of the anti-ageing effects of calorie restriction in skeletal and cardiac muscle. Int J Mol Sci. 2018; 19(4), doi: 10.3390/ijms19040928, indexed in Pubmed: 29561771.

5. Zecchin A, Pattarini L, Gutierrez MI, et al. Reversible acetylation regulates vascular endothelial growth factor receptor-2 activity. J Mol Cell Biol. 2014; 6(2): 116-127, doi: 10.1093/jmcb/mju010, indexed in Pubmed: 24620033.

6. Hsieh HJ, Liu CA, Huang B, et al. Shear-induced endothelial mechanotransduction: the interplay between reactive oxygen species (ROS) and nitric oxide (NO) and the pathophysiological implications. J Biomed Sci. 2014; 21: 3, doi: 10.1186/1423-0127-21-3, indexed in Pubmed: 24410814.

7. Huang B, Chen CT, Chen CS, et al. Laminar shear flow increases hydrogen sulfide and activates a nitric oxide producing signaling cascade in endothelial cells. Biochem Biophys Res Commun. 2015; 464(4): 1254-1259, doi: 10.1016/j.bbrc.2015.07.115, indexed in Pubmed: 26212441.

8. Liu J, Bi X, Chen T, et al. Shear stress regulates endothelial cell autophagy via redox regulation and Sirt1 expression. Cell Death Dis. 2015; 6: e1827, doi: 10.1038/cddis.2015.193, indexed in Pubmed: 26181207.

9. Wu LH, Chang HC, Ting PC, et al. Laminar shear stress promotes mitochondrial homeostasis in endothelial cells. J Cell Physiol. 2018; 233(6): 5058-5069, doi: 10.1002/jcp.26375, indexed in Pubmed: $\underline{29219180 .}$.

10. Go YM, Son DJu, Park H, et al. Disturbed flow enhances inflammatory signaling and atherogenesis by increasing thioredoxin-1 level in endothelial cell nuclei. PLoS One. 2014; 9(9): e108346, doi: 10.1371/journal.pone.0108346, indexed in Pubmed: 25265386.

11. Wu JM, Wang ZR, Hsieh TC, et al. Mechanism of cardioprotection by resveratrol, a phenolic antioxidant present in red wine (Review). Int J Mol Med. 2001; 8: 3-17.

12. Xia N, Förstermann U, Li H. Resveratrol and endothelial nitric oxide. Molecules. 2014; 19(10): 1610216121, doi: 10.3390/molecules191016102, indexed in Pubmed: 25302702.

13. Chen T, Li J, Liu J, et al. Activation of SIRT3 by resveratrol ameliorates cardiac fibrosis and improves cardiac function via the TGF- $\beta /$ Smad3 pathway. Am J Physiol Heart Circ Physiol. 2015; 308(5): H424H434, doi: 10.1152/ajpheart.00454.2014, indexed in Pubmed: 25527776. 
14. Lin MC, et al. Hsing, CH, Li, FA, Rosuvastatin modulates the post-translational acetylome in endothelial cells. Acta Cardiol Sin. 2014; 30: 67-73.

15. Wiese S, Reidegeld KA, Meyer HE, et al. Protein labeling by iTRAQ: a new tool for quantitative mass spectrometry in proteome research. Proteomics. 2007; 7(3): 340-350, doi: 10.1002/pmic.200600422, indexed in Pubmed: 17177251.

16. Chen Z, Peng IC, Cui X, et al. Shear stress, SIRT1, and vascular homeostasis. Proc Natl Acad Sci U S A. 2010; 107(22): 10268-10273, doi: 10.1073/pnas.1003833107, indexed in Pubmed: 20479254.

17. Klinge CM, Blankenship KA, Risinger KE, et al. Resveratrol and estradiol rapidly activate MAPK signaling through estrogen receptors alpha and beta in endothelial cells. J Biol Chem. 2005; 280(9): 7460-7468, doi: 10.1074/jbc.M411565200, indexed in Pubmed: $\underline{15615701 .}$

18. Cappello F, Zummo G. HSP60 expression during carcinogenesis: a molecular "proteus" of carcinogenesis? Cell Stress Chaperones. 2005; 10(4): 263-264, doi: 10.1379/14661268(2005)10[263:hedcam]2.0.co;2, indexed in Pubmed: $\underline{16333979}$.

19. Xu Q, Schett G, Seitz CS, et al. Surface staining and cytotoxic activity of heat-shock protein 60 antibody in stressed aortic endothelial cells. Circ Res. 1994; 75(6): 1078-1085, doi: 10.1161/01.res.75.6.1078, indexed in Pubmed: 7525102.

20. Hochleitner BW, Hochleitner EO, Obrist P, et al. Fluid shear stress induces heat shock protein 60 expression in endothelial cells in vitro and in vivo. Arterioscler Thromb Vasc Biol. 2000; 20(3): 617623, doi: 10.1161/01.atv.20.3.617, indexed in Pubmed: 10712382.

21. Shi L, Tu BP. Acetyl-CoA and the regulation of metabolism: mechanisms and consequences. Curr Opin Cell Biol. 2015; 33: 125-131, doi: 10.1016/j.ceb.2015.02.003, indexed in Pubmed: 25703630.

22. Infantino V, Iacobazzi V, Palmieri F, et al. ATP-citrate lyase is essential for macrophage inflammatory response. Biochem Biophys Res Commun. 2013; 440(1): 105-111, doi: 10.1016/j.bbrc.2013.09.037, indexed in Pubmed: 24051091.

23. Doddaballapur A, Michalik KM, Manavski Y, et al. Laminar shear stress inhibits endothelial cell metabolism via KLF2-mediated repression of PFKFB3. Arterioscler Thromb Vasc Biol. 2015; 35(1): 137-145, doi: 10.1161/ATVBAHA.114.304277, indexed in Pubmed: 25359860.

24. Zhang Y, Cui G, Wang Y, et al. SIRT1 activation alleviates brain microvascular endothelial dysfunction in peroxisomal disorders. Int J Mol Med. 2019; 44(3): 995-1005, doi: 10.3892/ijmm.2019.4250, indexed in Pubmed: $\underline{31257461 .}$.

25. Ballermann BJ, Dardik A, Eng E, et al. Shear stress and the endothelium. Kidney Int Suppl. 1998; 67: S100-S108, doi: 10.1046/j.1523-1755.1998.06720.x, indexed in Pubmed: $\underline{9736263}$. 
Figure 1. Protein acetylation regulated by shear flow and resveratrol; A. The Human umbilical cord vein endothelial cells (HUVECs) were coated on glass slides for either static (Control) or shear flow (SF, 12 dynes/cm ${ }^{2}$ ) treatments for 1 hour. Resveratrol (RSV, $10 \mathrm{uM}$ ) was added into a culture medium for 1 hour; B. Forty micrograms of protein were extracted from static, SF and RSV treatments were subjected to western blotting with anti-acetyl lysine monoclonal antibody (1:500). The expressions of acetylated proteins with significant differences from three repeated trials were indicated $(\mathbf{a}-\mathbf{f}) ; \mathbf{C}$. The relative fold-changes were shown as the mean \pm standard error. The changes were individually compared to the control by the Fisher LSD for significant difference $(\mathrm{p}<0.05)$; NS — no significance.

A
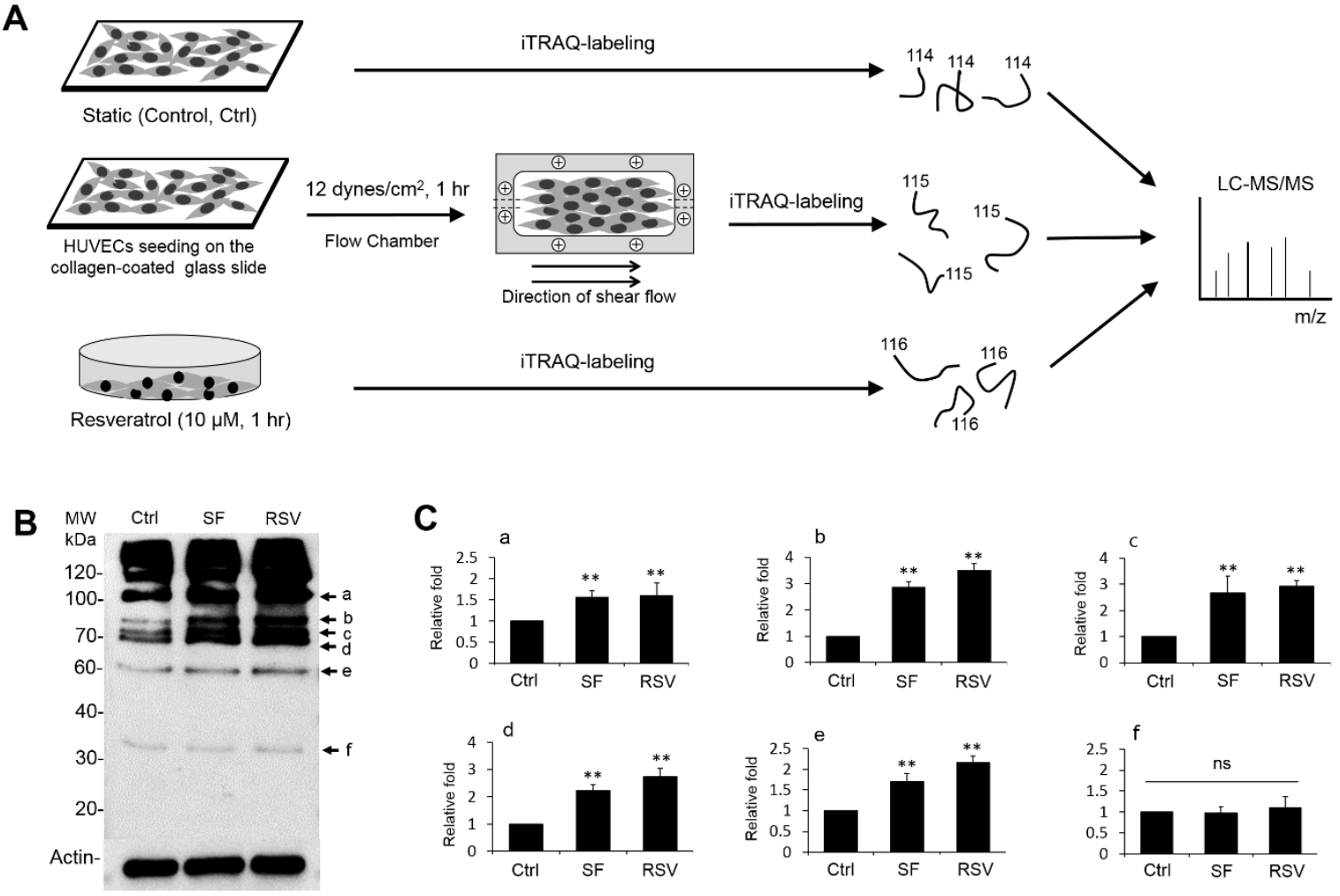
Figure 2. Acetylomes of lysine residues identified by liquid chromatography tandem-mass spectrometry (LC-MS/MS); A. The proteins with increased or decreased acetylation between shear flow (SF) and resveratrol (RSV) treatments were identified. The acetylated/deacetylated proteins co-regulated by two treatments were also indicated; B. The co-regulated acetylated proteins were analyzed by ingenuity pathway analysis software and five putative signalings were predicted.

A

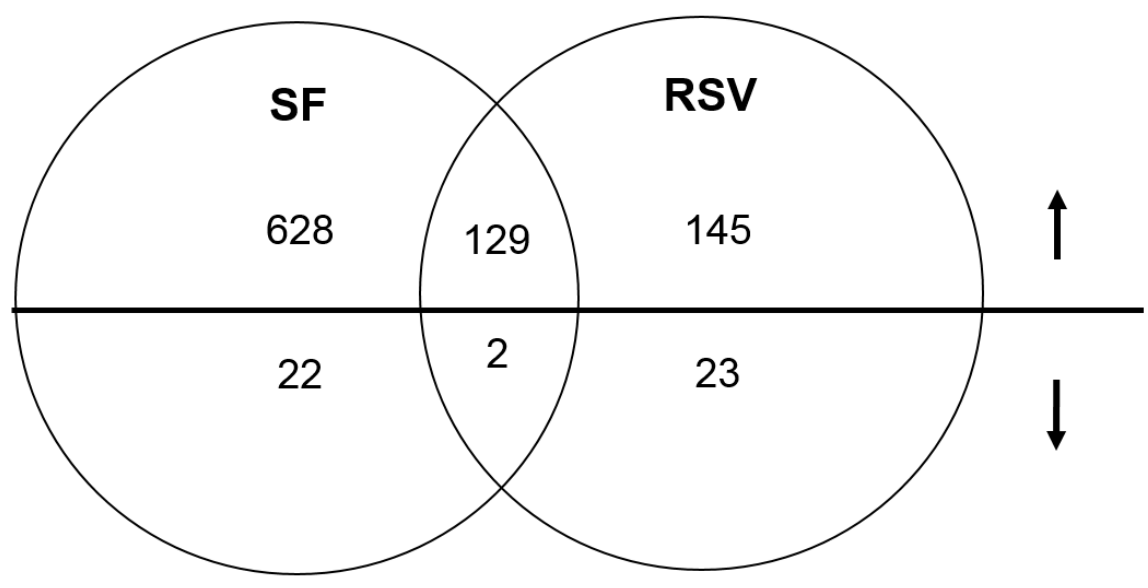

B

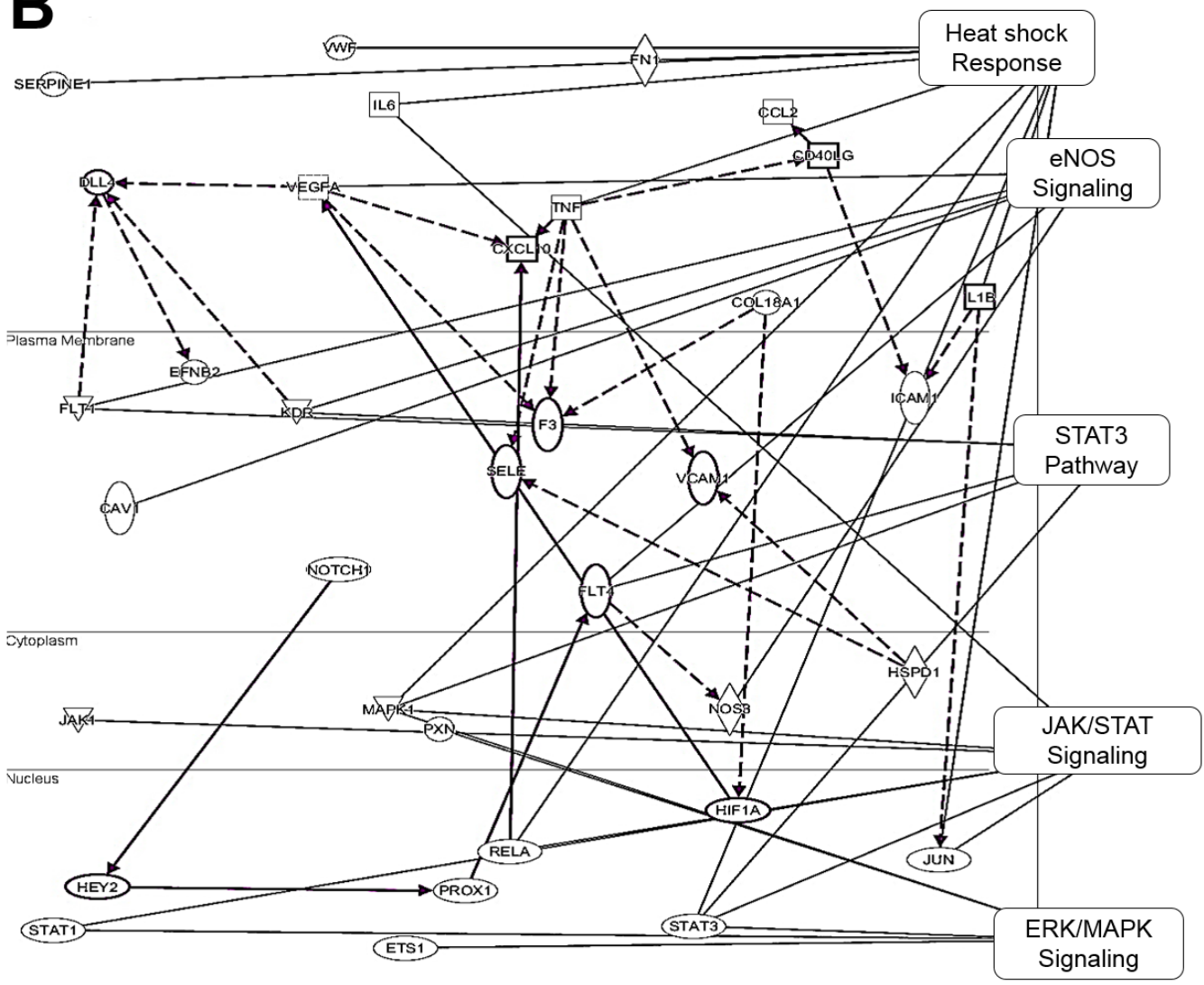


Figure 3. Algorithms of acetylated proteins. The putative acetylated lysine residues of eight proteins were indicated. The relative fold changes of acetylation levels between control, shear flow (SF) and resveratrol (RSV) were statistically shown from three repeat trials as means \pm standard error. The changes were individually compared to the control by the Fisher LSD for significant difference $(\mathrm{p}<0.05)$.

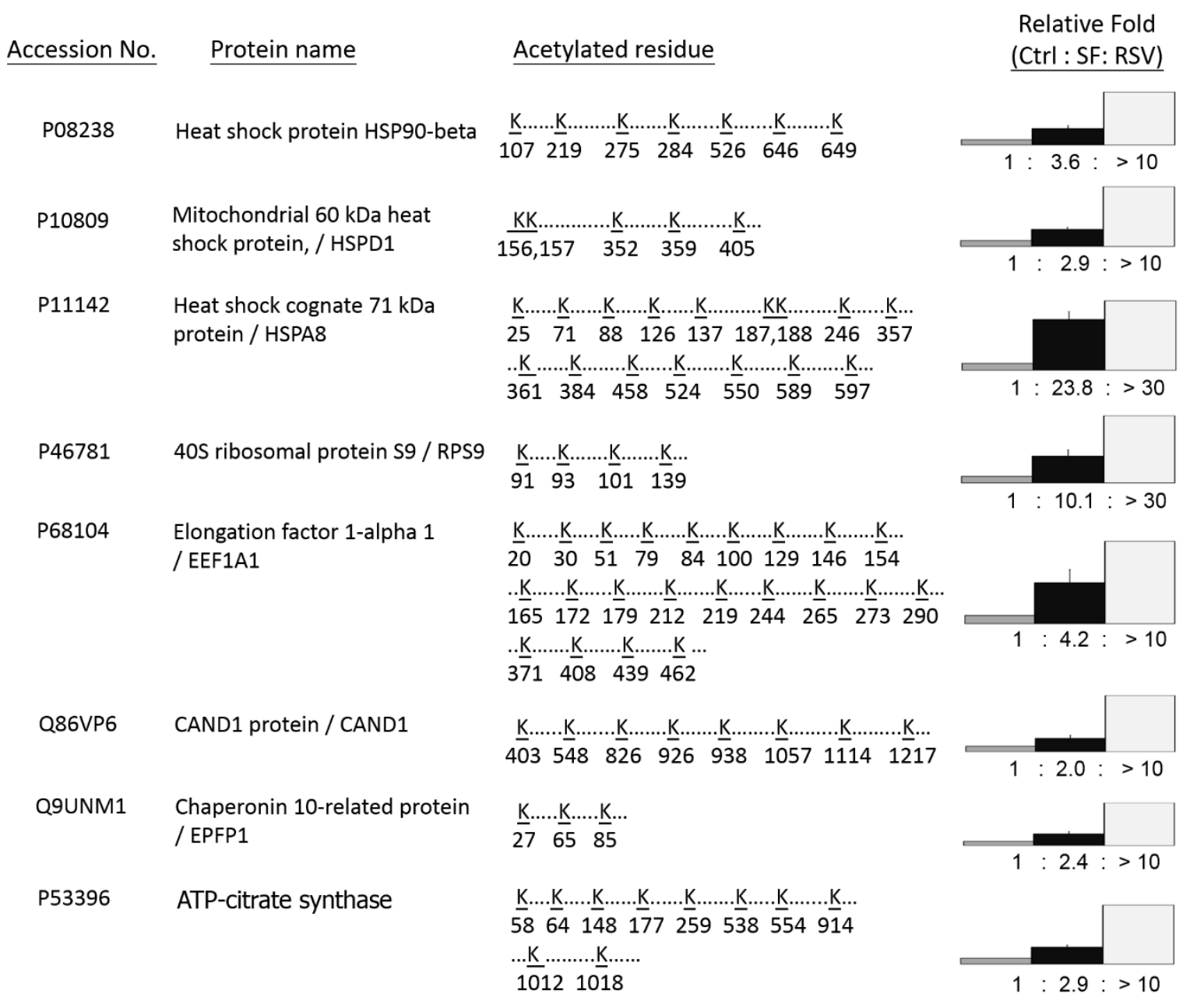


Figure 4. Verifications of protein acetylation; A. The whole cell lysates and the immunoprecipitation eluents obtained from three treatments were western blotted with HSP 90 (1:1000), HSP 60 (1:1000) and ATP-citrate synthase (1:1000) antibodies; B. The relative folds of acetylated protein/whole cell lysate were statistically calculated from three repeated trials as mean \pm standard error. The changes were individually compared to the control by the Fisher LSD for significant differences $(\mathrm{p}<0.05)$.
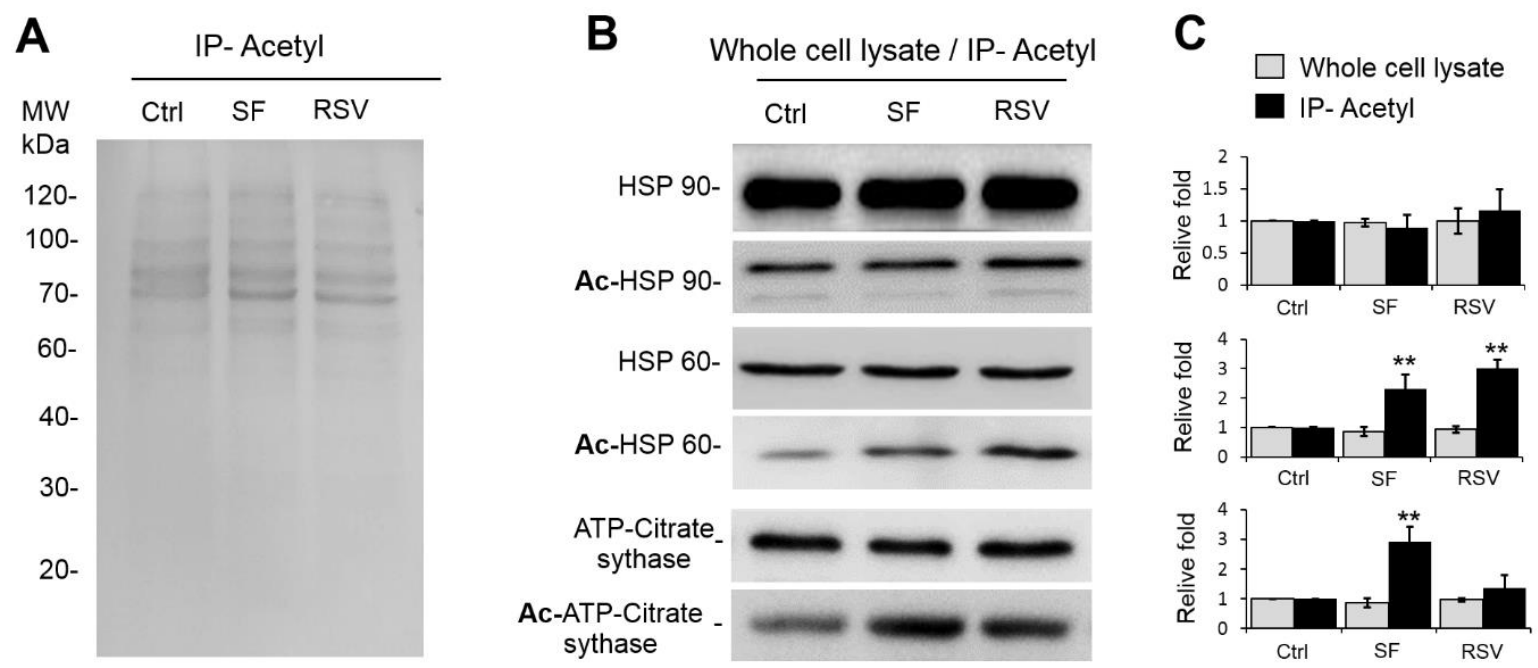